BNL -39787

$$
00 N+86091+a-5
$$

DE87 010438

\title{
EXPERIMENTING WITH LANGEVIN LATTICE QCD
}

\author{
R.V. GAVAI, J. POTVIN and S. SANIELEVICI* \\ Physics Department \\ Brookhaven National Laboratory \\ Upton, NY 11973
}

\begin{abstract}
We report on the status of our investigations of the effects of systematic errors upon the practical merits of Langevin updating in full lattice QCD. We formulate some rules for the safe use of this updating procedure and some observations on problems which may be common to all approximate fermion algorithms.
\end{abstract}

* Talk given by S. Sanielevici at Lattice Gauge Theory '86, BNL, 14-19 September 1986, to be published in the Proceedings.

This manuscript has been authored under contract number DE-AC02-76CH00016 with the U.S. Department of Energy. Aecordingly, the U.S. Government retains a non-exclusive, royalty-free license to publish or reproduce the published form of this contribution, or allow others to do so, for U.S. Government purposes. 
EXPERIMENTING WITH LANGEVIN LATTICE QCD

\section{S. SANIELEVICI}

Physics Department

Brookhaven National Laboratory

Lpton, NY 11973, LSA

in coliaboration with:

R.V. GAVAI and J. POTTIN

Broolinaven National Labozatory

$A B S T R A C T$

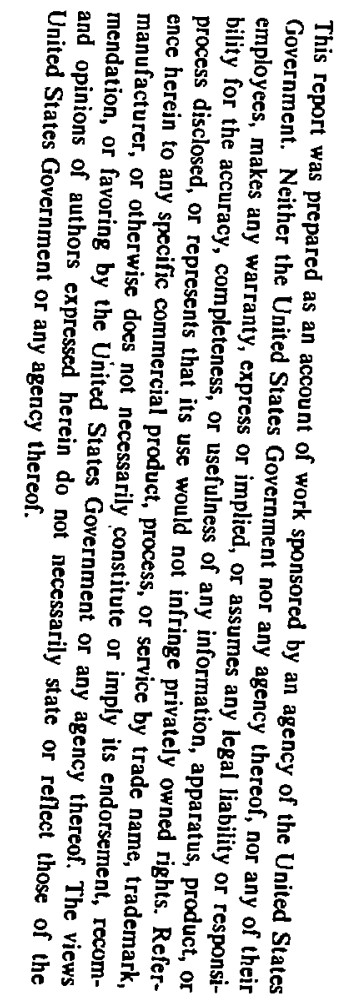

We report on the status of our investigations of the eñects oi systeratic errors upon the practical merits of Langevin upcating in full latice QCD. We formulate some rules for the saie use of this upiating procecure anc some observations on problems which may be common to all approx:mate fermion algotitins.

\section{NZRODUCEION AND ELMMARY}

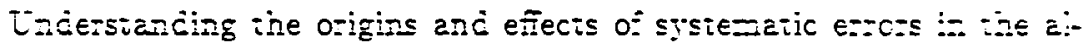
gorithms used fo: the numetica! simulation of latice $Q C D$ is hateasing: recogrizec as one of the major problems in ine neld I1. The upaaing procectre based on the discretized version of the Iangerin ectution in- is well suited fo: such silucies, because the origin of its systematic s:tozs is $w \in ! l$

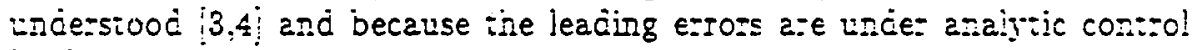

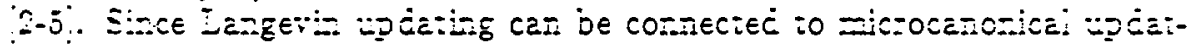
in

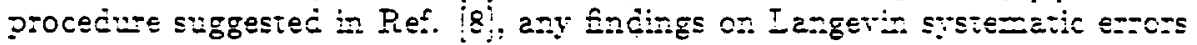

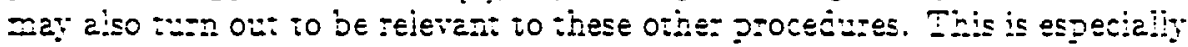
iikeiy io: ine specisic ễects of ine iermion deierminant. 
Our numerical study of the systematic errors in Langevin algorithms for QCD with dynamical quarks $[9,10]$ is restricted to basic (non-hybrid, real-action, non-Fourier-accelerated) updeting. It takes place on a $4^{4}$ lattice having periodic boundary conditions in $i$ directions and antiperiodic ones in the fourth. The coupling is fixed to $\beta=4.8$; we simulate four flavors of Kogut-Susskind quarks of masses $m a=0.1$ and $m a=0.05$ using the Cornell group's bilinear noise approach [2].

We measure planar Wilson loops at various values of the discrete step size in Langevin time. The systematic error originates in the finite value of this step size and becomes worse as the step size increases. It is interesting to assess the effect of the bias upon planar Wilson loops, because these are the fundamental building blocks of most lattice observables. Therefore, the errors on the fundamental Wilson loops will in general propagate nontrivially to plague the eventual physical quentities one wants to extract.

As a bencimark to assess the efiects of the error, we use the measurements of tise same loops peziomed with an algotithm which computes the fermion determinan directiy [11]. We aiso compare the Langevin resulis to inose ojiained with the pseudo-fermion metnod at rarious acceptances i11!. Since the numbe: of iterations necessary tor equilibration and the size of time correletions in equilibricm are imporicht in determining ine total cosi of a numerical expe-iment which aims for a given statistical erroz, we also zonito: these quantities by vatious methocis. Note that inese quantities decrease as tine : ime siep increases. What we recuire is a procecure which allows us to run ai ine la:gesi possible step size with an accepiable systematic erroz.

The aa:s result we want to present here is a set of "safery sules" to: jasgevis upeaing:

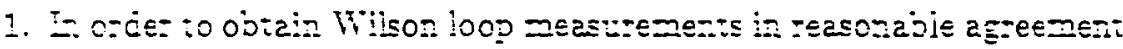

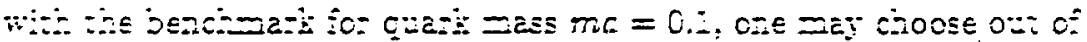
in-es me:inocis:

A. One iun with the firsi-orce: (Evle:) algctithm wing a the siep $\in$ S $0.00 \mathrm{i}$. Since time comeiations are rethe large with isis metinod, the run musi be suncientiy long.

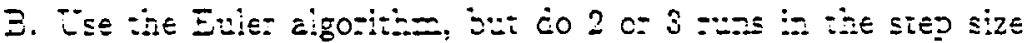

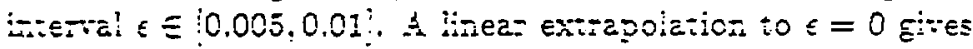
a gooć central ralue $\hat{10}$ your loop.

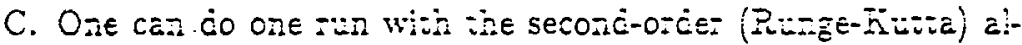

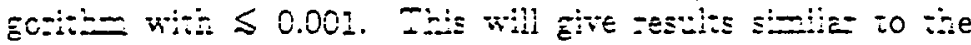


ones obtained by method $\mathrm{A}$; time correlations will be smalier but the overall cost is slightly larger because this algorithm requires two conjugate gradient inversions instead of one.

2. For the values of the step size mentioned above, the non-integrable contribution to the leading systematic error is numerically unimportant.

3. There are physically important problems of a qualitative nature, such as the presence or absence of a first-order phase transition, which can be investigated without worrying about the systematic error. One can study these using either first order or second order algorithrns, with a step size (say, $\epsilon=0.01$ ) which should still be small enough that the absolute values of the monitored quantities (plaquette, $\bar{x} X$, Polyakov line) are reasonable. When using the Euler a!goritnm, one should shift the coupling: number of fiavors anc mass according to the formulae given in Reiss. 20.5,10:

4. The effect of the systematic erro: becomes more severe when the ciuark mass is decreasec to $m c=0.05$. In this case, runs at $\epsilon=0.001$ are no longe: süfincient: one shoulè go io still smailer siep sizes.

\section{ALGORITHMS}

We shal: begin by briefy reviewing the basic notations anc formalism of ciscreie iangevin difinusion processes in SU(3) group space, ine o:igin of systematic errors in Langevin upaiting and the algorithms we have been

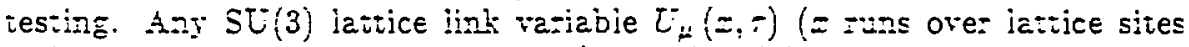

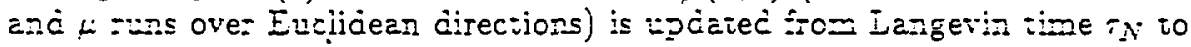
$T_{-2}$ by ine formula

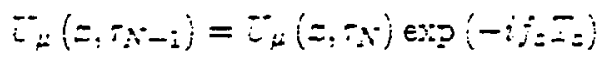

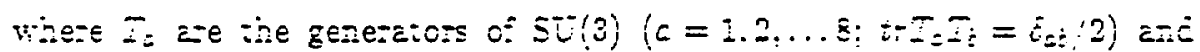
$i=t i t, r_{i} \dot{i}$ is called the c-iving force. The criving force is built out

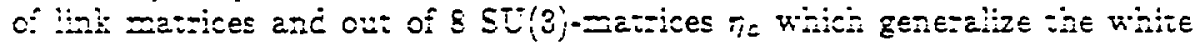

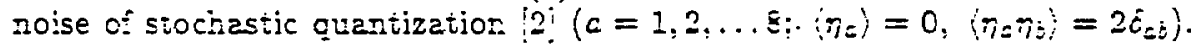

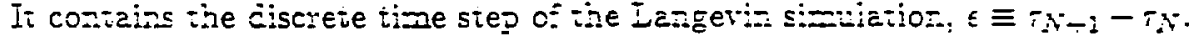

An given choice of the criving force cennes a Ianger: up iating aigonth= fo: ine ineory. One cen inen use ine Eo

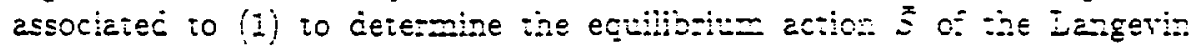

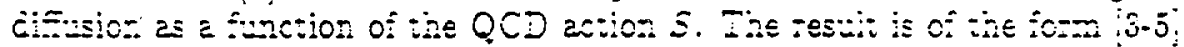

$$
\bar{S}=S+o\left(\epsilon^{\pi}\right): \quad(\pi=1,2, \ldots)
$$


This difference between $\bar{S}$ and $S$ is the origin of the systematic ex:s: in Langevin updating. The explicit form of the relationship (2) can be used to engineer $f_{c}$ such that the error be minimal ( $n$ in Eq. (2) maximal). Unfortunately, (2) can be worked out analytically (so far) only to first order in $\epsilon$, so that algorithms can only be improved to yield $n=2$ [2-5]. Therefore, numerical experiments remain the only way to assess the effects of the systematic bias upon lattice observables.

We report here on the performance of the following Langevin algorithms for. full $Q C D$ :

1. First-order (Euler) QCD with fermionic noise. The driving force is [3]

$$
f_{a}=\epsilon\left(\partial_{a} S_{g}[U]-\frac{1}{4} \xi^{\dagger} A_{a}[U] \xi\right) \div \sqrt{\epsilon} \eta_{a}
$$

where $S_{g}$ is the usual Milson action,

$$
A_{c}[U]=M^{-1}[U] \partial_{c}\left(M^{\dagger} M\right) M^{-1}[U]
$$

$M=D-$ ma such that $S=S_{t}-(1 / 2) T: i=M^{i} M$ for 4 fiaro:s 0 : siaggered quarks and $\xi$ is a bilinea: no.se, no:mailized like ine $\eta_{a}$ 's. This leaves $\bar{\Sigma}-S$ of orcier ố order $\epsilon$ \3,5!

2. "Najve" second-order (Rung-Kuita) QCD with iermionis noise. This is den̂ned by the d-iving force !5]

$$
\begin{aligned}
& \therefore=\frac{\epsilon}{2}\left(1-\frac{C_{2} \epsilon}{12}\right)\left(\partial_{a} S_{s} i \tau:-\partial_{s} S_{z}: \tilde{U} ;\right.
\end{aligned}
$$

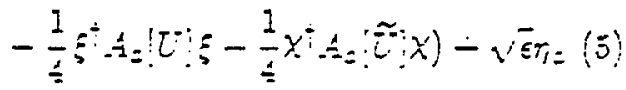

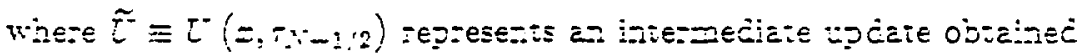
by Ess. (1) and (3) and $x$ is a second bitinea: noise. The

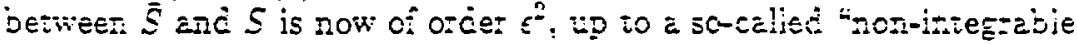

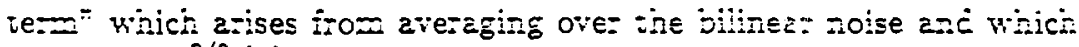
is of order $\epsilon^{3 / 2}$ is:

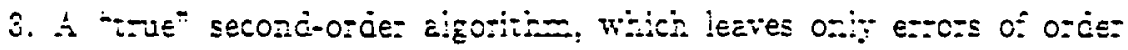

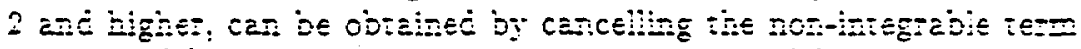
explictity 5. . This is cone by zeplacing $r_{i}:=$ Ec. (5) oy

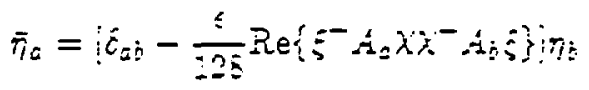




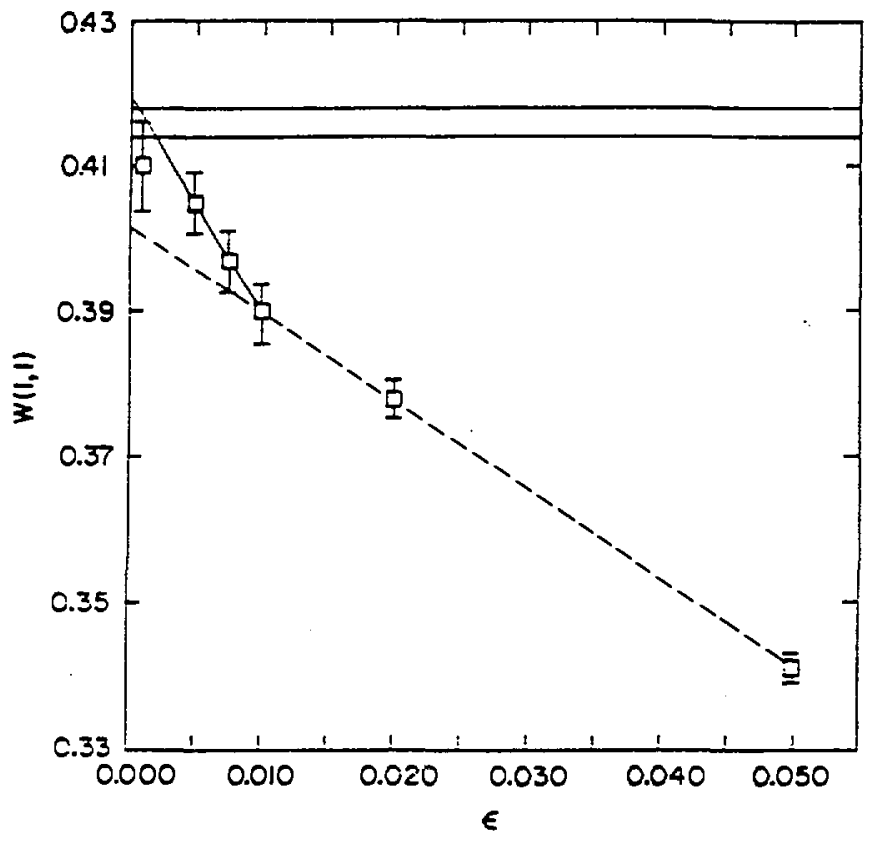

Fig. 1: Periormance of the Euler algorithm for Langevin QCD. The full corridor indicates the benchmark result [11]. Error bars are corrected for time correlations in equilibrium [12]. Straight ines represent possible exirapolations.

\section{TEE ETIER ALGORITEM}

Figure 1 presents the dependence of the $1 \times 1$ hiison ioop on ine siep size. It has been obtained by using the Euler algorithm unde: the following concitions: $3=6 / g^{2}=4.8$, the oua-k mass in latice units $m a=0.1$, four favors of Kogut-Sussicind quarks. The inversion of $M$ was done by conjugate gradien:. We imposed the stopping condition $r=\left(\| M \bar{z}-\overline{s i i^{2}}\right)^{1 / 2}<0.6 \overline{\text {. }}$ Noie tinat our definition of the resiaue differs from othe: popula: defnitions by not civicing out the length of the vector $\bar{\xi}$ (our $T=0.05$ is of the order $10^{-5}$ in the othe: normalization). In the mear, the recuired aceuract was reacined afte: 65 conjugate gracien iterations. The upating ime per ink 
resulted to be about 0.88 milliseconds on the CRAY X-MP 22 at NMFECC (as compared to about 0.77 milliseconds for a similarly optimized pseudofermion code). Equilibration was checked in all cases by comparing averages over successive batches of 3000 iterations, for all Wilson loops up to $3 \times 3$. At $\epsilon=0.01$ we also checked that hot and cold starts converge to the same average plaquette. The final averages were then obtained over 3000 iterations at equilibrium.

The corridor in Fig. 1 represents the result of the recent direct computation of the fermion determinant [11], which was done under exactly the same conditions as the present study. We see that we can come close to the benchmark either by running at sufficiently low $\epsilon(\epsilon \leqslant 0.001)$ or by doing two runs in the interval $\epsilon \in[0.005,0.01]$ and extrapolating linearly to $\epsilon=0$. An extrapolation based on runs below $\epsilon=0.01$ would underestimate the value of the plaquette by far.

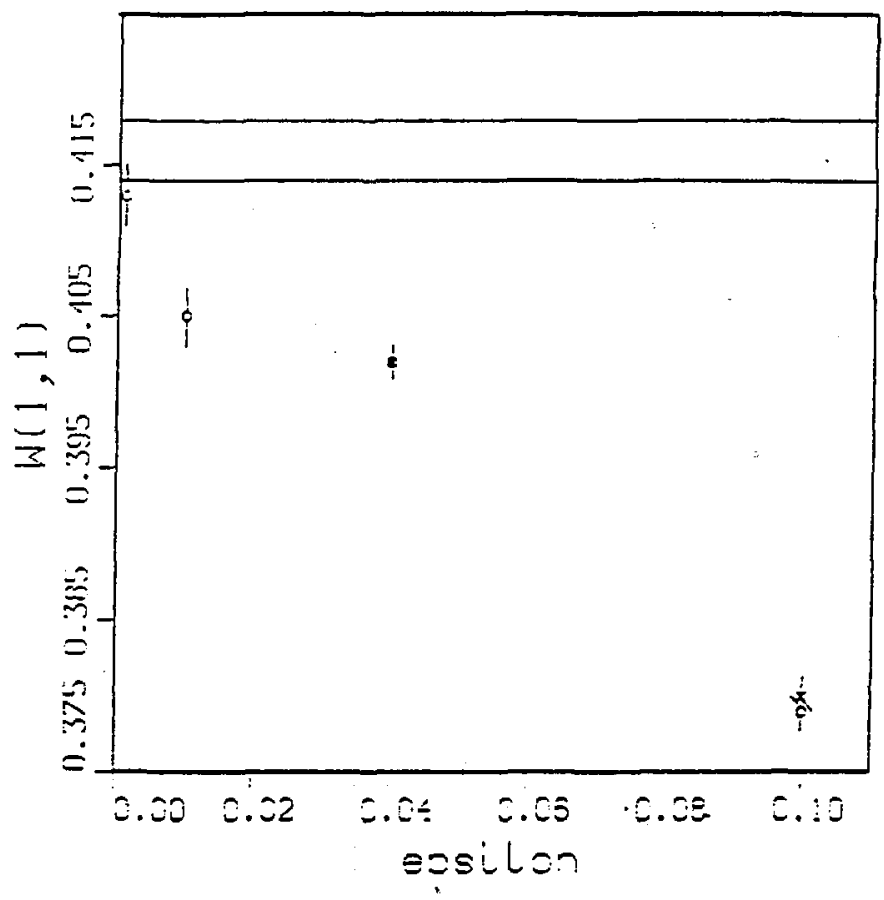

Fig. 2: Periormance of Runge Kutia algorithms fo: Langevin QCD. Open circles represent the resulis of the aigoriting of Ec. (5) anc crosses

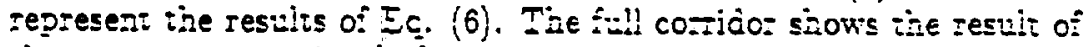
the exact computation [11i. 
TABLE I: Relative performance of Langevin and pseudofermion algorithms ( $m a=0.1$ ). Algorithm $D$ is the direct computation [11], $P$ reproduces the relevant pseudo-fermion results of $[11]$ and $L$ is the Runge-Kutta algorithm of (5).

\begin{tabular}{|c|c|c|c|c|c|c|c|c|}
\hline Alg. & & Acc. & $W(1,1)$ & $W(1,2)$ & $W(1,3)$ & $W(2,2)$ & $W(2,3)$ & $W(3,3)$ \\
\hline D & & & .042 & .039 & .023 & .016 & .005 & .001 \\
& & & $(.003)$ & $(.003)$ & $(.003)$ & $(.003)$ & $(.002)$ & $(.002)$ \\
\hline P & & .86 & .042 & .039 & .024 & .017 & .005 & .001 \\
& & & $(.002)$ & $(.002)$ & $(.001)$ & $(.001)$ & $(.001)$ & $(.001)$ \\
\hline L & .001 & & .039 & .038 & .024 & .018 & .004 & .003 \\
& & & $(.002)$ & $(.002)$ & $(.002)$ & $(.002)$ & $(.002)$ & $(.002)$ \\
\hline P & & .74 & .030 & .027 & .016 & .010 & .003 & .001 \\
& & & $(.002)$ & $(.002)$ & $(.001)$ & $(.001)$ & $(.001)$ & $(.001)$ \\
\hline L & .01 & & .031 & .029 & .018 & .011 & .002 & .002 \\
& & & $(.002)$ & $(.002)$ & $(.001)$ & $(.001)$ & $(.001)$ & $(.001)$ \\
\hline
\end{tabular}

\section{RUNGE-KUTTA ALGORITHMS}

Figure 2 contains the same information as Figure 1 for the case of the Runge-Kutta algorithms (5) and (6). Table I assesses the quality of various planar Wilson loops measured using Eq. (5) with res act to the benchmark set in Rei. [11] and with respect to the pseudo-fermion algorithm at various acceptances [11]. Uising Eq. (5), one must do 2 conjugate gradient inversions per Langevin step; the updating time per lint becomes about 1.65 miliiseconds and the memory. requirement becomes about $5 / 4$ that for the Fule: o: pseucio-fermion-schemes. Eq. (6) requires 3 inversions pe: time siep inc iakes aboui 2.37 miliiseconds per link upáate.

We see that the higher-order errors are still substantial unless $\epsilon \lessgtr$ 0.001 , in which case boin îrst-and higher-orcer ercors are zather small. The improvement gained by canceliing the non-iniegrable term is seen to be insignificani. There appears to be a correpondence between the Langevin and the pseudo-fermion algoritims: Runge-Kutta at $\epsilon=0.001$ is comparable to psevio-fe:mions at $86 \%$ acceptance and Runge-Kutia ai $\epsilon=0.01$ to pseucioît:Dions at $i 4 \pi$ accepiance. Time correiations anc hence int correctec siatistjcal e:rors [12] are systematically smalle: fo: the Runge- Huita than fo: the Euler scheme. However, the total run time reouired to achieve a given accuracy would still be highe: for the second-orde- scheme because of the adaitional matrix inversion. 


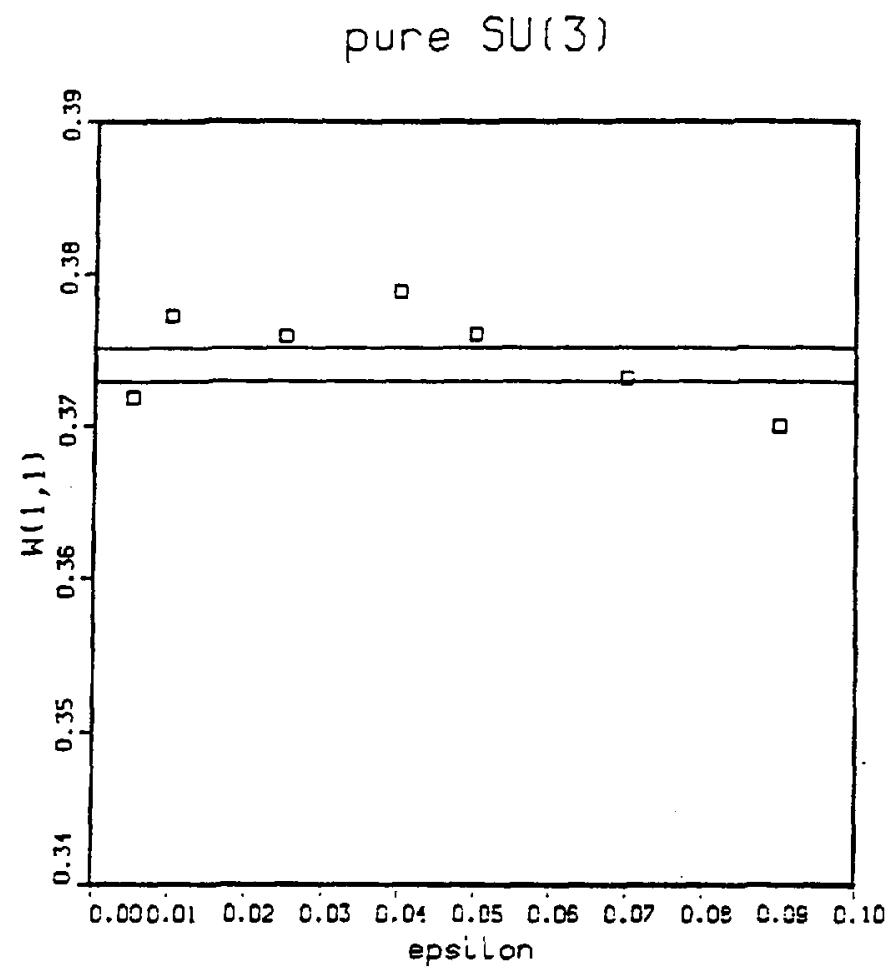

Fig. 3: The Runge-Kutta algorithm for Langevin pure SU(3). All the runs are on a $4^{4}$ lattice with $\beta=4.8$. The benchmark is based on runs with the standard Metropolis et al. method.

\section{LOWERING THE QUARK MASS}

Ref. [11] has observec that the pseudo-fe:mion algo:itisn performs less well with respect to the bencinmari at ouari mass ma $=0.05$ than at $m a=0.1$. Would this also be the case fo: Langevin updaing? We have run tine Runge-riutta algoritim Dased on Eq. (5) at $m a=0.05$ in order to answe: this cuestion. Lising $\epsilon=0.001$ and a stopping resicue of 0.2 (as in [11]) which is reached atter 121 conjugate gradient iterations on the average, we spend about 2.13 milliseconds pe: link update and find

$$
\langle W(I, I)\rangle=0.404 \doteq 0.003
$$

as compared to [11]

$$
\begin{array}{cc}
\langle W(1,1)\rangle=0.417 \doteq 0.002 & \text { (DIRECT ALGORITHM) } \\
\langle W(1,1)\rangle=0.410 \doteq 0.006 & \text { (PSEUDO - FERMIONS 86\%) }
\end{array}
$$


We tried lowering the stopping residue to $r=0.01$ (reached after about 189 conjugate gradient iterations: 3.32 milliseconds per link update) but all the Wilson loops remained the same within errors (for instance, $\langle W(1,1)\rangle=$ $0.405 \pm 0.003$ ).

It would seem that Langevin at $\epsilon=0.001$ is still comparable to pseudo- fermions at $86 \%$ acceptance and that the effective systematic error increases for both algorithms as the quark mass is decreased. That this is indeed an effect of the non-linear fermion determinant can be seen by comparing Fig. 2 to Fig. 3, which shows the $\epsilon$-dependence of $V^{\prime}(1,1)$ in pure SU(3) [10]. The range of $\epsilon$ where Langevin algorithms give good results appears to shrink progressively as the quark mass decreases from infinity. This tends to confirm the observation [13] that the residual systematic error in $\mathrm{Eq}$. (2), for fermionic schemes, is actually of the order $\epsilon^{2} / \lambda^{4}$, where $\lambda$ is some average over field configurations of the lowest eigenvalue of the larifice Dirac operator. It is known that this average decreases with the quarl mass. It is tempting to speculate that the same effect is also seen in the pseudofermion scheme.

\section{CONCLUSIONS}

Our numerical experiments have shown that the analytically intractable terms of order $\epsilon^{2}$ and higher in Eq. (2) have substantial effects upon planar Wilson loops in full QCD. Based on the known fist-ordez correction terms, one had hoped that all systematic errors could either be eliminated by improving the naive Euler algorithm or they would be irrelevant in the continuum limit. Even though the quantitative insignificance of the leaaingorder non- integrable term is good news in this respect, the large effects oi highe:- order terms mean that such an optimistic conciusion cannot be taken frr granted in the general case. One should therefore respect the isafery "uies" we recommended above.

A study such as the present one obriousig ceperis voon ihe exisience of reliable and accurate benchmarks. It is therefore vey imporiant to pursue

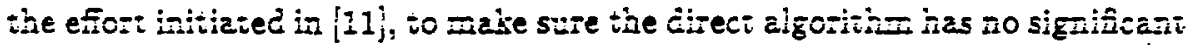
hidden bias of its own and to improve the statisics of these bencina-is rids.

Acirnowiledgment

This work was supporied by tine Ü.S. Departmeni of Energ: linder contract number DE-AC02-76CH00016. The present autioo wishes to tinan: ine Natural Sciences arc Engineering Research Council of Canacia fo: anarcizi suppor:. 


\section{REFERENCES}

1. D. Weingarten, Nucl. Phys. B257 [Fs 14](1981) 629.

2. G. Parisi and Y.S. Wu, Sci. Sin. 24 (1981) 483.

3. G.G. Batrouni, G.R. Katz, A.S. Kronfeld, G.P. Lepage, B. Svetitsky, and K.G. Wilson, Phys. Rev. D32 (1985) 2736.

4. A. Ukawa and M. Fukugita, Phys. Rev. Lett. 55 (1985) 1854.

5. G.G. Batrouni, Phys. Rev. D33 (1986) 1815;

A.S. Kronfeld, Phys. Lett. B172 (1986) 93.

6. See e.g. J.B. Kogut, Nucl. Phys. B270 [FS16](1986) 169.

7. F. Fucito, E. Marinari, G. Parisi, and C. Rebbi, Nucl. Phys. B180 [FS 2](1981) 369.

8. M. Creutz and R.V. Gavai, BNL-38204 (1986), to appear in Nucl. Phys. $\mathrm{B}$.

9. R.V. Gavai, J. Potvin, and S. Sanielevici, BNL-38526 (1986), to appear in Phys. Lett. B.

10. R.V. Gavai, J. Potvin, and S. Sanielevici, in preparation.

11. R.V. Gavai and A. Gocksch, Phys. Rev. Lett. 56 (1986) 2659;

R.V. Garai, A. Gocksch, and U.M. Heller, BNL-38449 and NSF-ITP86-89 (1986); to appear in Nucl. Phys. B.

12. K. Binder, in "Phase Transitions and Critical Phenomena", C. Domb and M.S. Green ecs., vol. 5B (Academic, New Yo:k, 1976).

13. M. Fukugita, these Proceedings. 